\title{
Surface-modified poly(lactide-co-glycolide) nanospheres for targeted bone imaging with enhanced labeling and delivery of radioisotope
}

\author{
Yoon Jeong Park, ${ }^{1}$ Sook Hee Nah, ${ }^{2}$ Jue Yeon Lee, ${ }^{2}$ Jae Min Jeong, ${ }^{3}$ Jun Key Chung, ${ }^{3}$ Myung Chul Lee, ${ }^{3}$ \\ Victor C. Yang, ${ }^{1}$ Seung Jin Lee ${ }^{2}$ \\ ${ }^{1}$ College of Pharmacy, The University of Michigan, 428 Church Street, Ann Arbor, Michigan 48109-1065 \\ ${ }^{2}$ Department of Industrial Pharmacy, College of Pharmacy, Ewha Womans University, 11-1 Daehyun-Dong, \\ Seodaemun-Ku, Seoul 120-750, Korea \\ ${ }^{3}$ Department of Nuclear Medicine, College of Medicine, Seoul National University, 28-2 Yongon-Dong, Chongno-Ku, \\ Seoul 110-820, Korea
}

Received 3 October 2002; revised 10 April 2003; accepted 30 April 2003

\begin{abstract}
Surface-modified nanospheres can be utilized for targeting drugs and diagnostic agents to the bone and bone marrow while extending their circulation time in the blood stream. The surface modification of poly(lactide-co-glycolide) (PLGA) nanospheres by radioisotope carrying poly(ethylene oxide)-poly (propylene oxide)-poly (ethylene oxide) triblock copolymers (Poloxamer 407) has been assessed by in vitro characterization and in vivo biodistribution studies after intravenous administration of the nanospheres to the mouse. A hydroxyphenylpropionic acid, a ligand for ${ }^{125} \mathrm{I}$ and ${ }^{131}$ I labeling, was conjugated to the hydroxyl group of the Poloxamer 407 by using dicyclohexyl carbodiimide. The ligand-conjugated Poloxamer 407 was adsorbed onto the surface of PLGA nanospheres. Surface coating was confirmed by measuring both size distribution and the surface charge of the nanospheres. Besides, ${ }^{125}$ I-labeling efficiency, radiolabeling stability, whole body imaging, and biodistri-
\end{abstract}

bution of the radioisotope-labeled nanospheres were examined. Ligand-labeled, surface-modified PLGA nanospheres were in 100-nm size ranges, which may be adequate for long-circulation and further bone imaging. ${ }^{125}$ I-labeling efficiency was $>90 \%$ and was more stable at human serum for $24 \mathrm{~h}$. A noticeable decrease in liver or spleen uptake was obtained by the surface-modified nanospheres. ${ }^{125} \mathrm{I}$-labeled nanospheres showed higher blood maintenance and bone uptake compared with stannous colloid with the same size distribution. Therefore, a fully biodegradable, radioisotopecarrying, surface-modified nanosphere system has been developed as a promising tool for targeting bone and bone marrows. (c) 2003 Wiley Periodicals, Inc. J Biomed Mater Res 67A: 751-760, 2003

Key words: polymer nanospheres; Poloxamer 407; radioisotope carrier; bone and bone marrow targeting; imaging

\section{INTRODUCTION}

Bone and bone marrow imaging may be potentially useful for prompt diagnosis of osteomyelitis, septic arthritis, and multiple myeloma. ${ }^{1-5}$ As well as diagnosing bone disease, imaging bone marrow can be useful for diagnosing cancer metastasis because it is a favored site for metastasis of many solid tumors. ${ }^{6-10}$ Recent studies have shown that several intravenously administered specific colloids can be taken up by bone marrow, allowing the improved detection of small bone tumors in an animal model. ${ }^{6-8}$ To this regard,

Correspondence to: S. J. Lee; e-mail: sjlee@ewha.ac.kr Contact grant sponsor: Nuclear Energy R\&D Program, The Ministry of Science and Technology

(C) 2003 Wiley Periodicals, Inc. specific and high-resolution imaging of bone and bone marrow can provide insight in determining the potency of disease, and can help in planning the treatment schedule. In addition, the success of imaging and following therapy depends on the critical relationship between the amount of radionuclides at the target tissue and the normal tissue. Small particles in nanometer ranges have been routinely used in many clinical diagnostic tests. As examples of bone and bone marrow imaging agents, ${ }^{99} \mathrm{~m}$ Tc-MDP, ${ }^{1,2}{ }^{99} \mathrm{~m}$ Tc-antimony colloid, ${ }^{1,2}{ }^{99} \mathrm{~m}$ Tc-sulfur colloid, ${ }^{3,5}{ }^{111}$ Indium colloid, ${ }^{2}$ and ${ }^{99 \mathrm{~m}} \mathrm{Tc}$-labeled human serum albumin nanocolloid $^{4,5}$ are currently used. Generally, requirements for a long-circulating bone imaging agent have been high specificity to the bone, optimal blood stability of radioisotope labeling, ability to avoid reticuloendothelial system (RES) uptake by the liver or spleen, low toxicity, and proper excretion. ${ }^{1-5,11-14}$ 
Bone marrow targeting has been attempted by using the recognizable molecule, such as transferrin ${ }^{15-17}$; however, targeting efficiency has been limited because the transferrin receptor also exists in the liver and spleen. The main hurdle for bone marrow targeting and imaging, thus, is that it is difficult to find a specific recognizable moiety by bone marrow surface endothelial system, because the bone marrow surface primarily has similar RES to liver or spleen except that bone marrow has more fenestrated RES than liver or spleen. A small fraction of the intravenously administered particulates reaches the bone and bone marrow, the next biggest RES than the liver and spleen, after removal by the RES of the liver and spleen. ${ }^{18,19}$ However, because bone marrow has the biggest RES after liver and spleen, passive direction after escaping liver/ spleen toward bone marrow has been the alternative to ferry the diagnostics to the bone marrow.

To avoid rapid liver/spleen RES uptake and obtain long circulation behavior, achieving proper particle size between 50-150 nm with a hydrophilic surface has been suggested. ${ }^{20,21}$ Unless the hydrophilic particles have the sugar moiety recognizable by hepatocytes in the liver, most nano-sized particles with hydrophilic polyethylene oxide (PEO) are considered to extend retention time in the circulation. Indeed, coating colloidal particles with PEO has been able to hinder the liver or spleen uptake and efficiently obtain a deposition of the particles primarily in the bone marrow. ${ }^{22,23}$ This effect is due to the steric repulsion of the PEO chain against both protein adsorption and particular macrophage adhesion by the liver or spleen. Marked redirection toward the bone followed by bone marrow uptake utilizing surface-modified particles with the PEO chain, as well as prevention of the liver/spleen uptake, has been demonstrated by several previous reports. ${ }^{20-23}$ Several hypotheses related to the interaction between PEO and bone marrow macrophage, although it is not yet clear, have been suggested to corroborate the improved bone uptake.

Herein, radioisotope-carrying, surface-modified poly(lactide-co-glycolide) (PLGA) nanospheres with poly(ethylene oxide)-poly(propylene oxide)-poly(ethylene oxide) (PEO-PPO-PEO) triblock copolymer (Poloxamer 407) were prepared for efficient targeted imaging of the bone and bone marrow. Nanospheres were made by routine $\mathrm{o} / \mathrm{w}$ emulsion/evaporation method. Poloxamer 407 coating was conducted by incubating ligand-labeled poloxamer solution with prepared nanospheres. Hydrophobic PPO unit can be anchored into the surface of nanospheres whereas PEO unit may increase the hydrophilicity of the surface of nanospheres. PEO units also induce repulsive steric hindrance, which interrupt the adhesion of the blood protein and uptake by RES of liver or spleen. Before incubation, PEO unit of the Poloxamer 407 was modified by ligand molecule that chelates radionuclide.
Hydroxyphenylpropionic acid (HPP), a ligand of radioactive ${ }^{125} \mathrm{I}$, ${ }^{131} \mathrm{I}$, was coupled to the hydroxyl group of PEO by using a coupling agent, dicyclohexyl carbodiimide (DCC). Ligand-conjugated Poloxamer 407 was coated onto the nanoparticles, and specific radionuclide to ligand, such as ${ }^{125} \mathrm{I}$ or ${ }^{131} \mathrm{I}$, was labeled to those surface-modified nanospheres before use in diagnosis. This unique method of labeling was expected to overcome the limitation of the current labeling method including physical entrapment into the nanosphere or direct coupling in respect to avoiding nonspecific leakage of isotope to the blood stream or loss of radioactivity of isotope. ${ }^{24,25}$ These ligand-conjugated polymers are most favorable for being ready to carry radioisotope in bone imaging. Radioisotopes could be easily chelated with ligand-copolymer conjugates in a short time, which permits immediate use for organ imaging and diagnosis. Also, the nanoparticulate carrier may permit simultaneous encapsulation of therapeutic drugs or gene vectors, thus providing a combined diagnostic and therapeutic bone/bone marrow drug delivery system.

Conjugation of radioisotope-specific ligand to Poloxamer 407, fabrication of nanospheres, surface modification, and characterization of surface-coated nanospheres, radioisotope labeling efficiency, stability, and biodistribution of the nanospheres will be discussed in this article.

\section{MATERIALS AND METHODS}

\section{Materials}

PLGA (MW 2000) was obtained from Polyscience Inc. (Warrington, PA). 3-(4-Hydroxyphenyl) propionic acid (HPP) was purchased from Aldrich Chemical Co. (Milwaukee, WI). DCC was supplied from Sigma (St. Louis, MO). Poloxamer 407 was obtained from BASF (Ladwingshafen, Germany). N-hydroxysuccinimide was from Fluka (Ronkonkoma, NJ). Methylene chloride and dimethylformamide (DMF) were purchased from Yakuri Pure Chemicals Co. (Osaka, Japan). Polyvinyl alcohol (PVA) was from Hayashi Pure Chemicals Ind. Ltd. (Osaka, Japan). ${ }^{125}$ I and ${ }^{131}$ I were obtained from Amersham Co. (Buckinghamshire, UK). All other chemicals were of analytical grade.

\section{Ligand conjugation with Poloxamer 407}

Fifty milligrams of HPP, as a ligand for iodine, was dissolved in $30 \mathrm{~mL}$ of dried DMF containing $30 \mathrm{mg}$ of DCC and $30 \mathrm{mg}$ of N-hydroxysuccinimide. This mixture was stirred for $2 \mathrm{~h}$ to activate carboxylic acid of HPP. Precipitants were removed by centrifugation. Then Poloxamer 407, dissolved in DMF, was added, and stirred for a further $4 \mathrm{~h}$ at room temperature. The product was precipitated in an excess of 
dry ether. Then the precipitate was dissolved in $20 \mathrm{~mL}$ of distilled water, dialyzed against distilled water (MWCO 1000), and freeze-dried. Formation of ligand-conjugated Poloxamer 407 was ascertained by using Fourier transform infrared spectrophotometer and differential scanning calorimetry.

\section{Nanosphere fabrication and surface modification of PLGA nanospheres by HPP-Poloxamer 407}

PLGA nanospheres were produced by an o/w emulsification/solvent evaporation technique involving dropwise addition of $30 \mathrm{~mL}$ of a solution of PLGA in methylene chloride to $300 \mathrm{~mL}$ of an aqueous, $1.5 \%(\mathrm{w} / \mathrm{v})$ solution of the PVA stabilizer. The concentration of the PLGA solution was $2 \%$. The PLGA solution was added over $1 \mathrm{~min}$ to the PVA aqueous solution, which was stirred at a rate of $8000 \mathrm{rpm}$ using a Silverson homogenizer (Silverson Machines, Chesham, UK), and stirring was continued for a further 10 min. These emulsions were heated at $85^{\circ} \mathrm{C}$ for $5 \mathrm{~min}$ and further stirred to remove methylene chloride. The resulting nanospheres were harvested and cleaned by repeated centrifuging and suspending in distilled water three times, then finally collected by lyophilization. The prepared nanospheres were further modified on their surface by incubating with HPP-Poloxamer 407 solution $(5 \mathrm{mg} / \mathrm{mL}$ ) for up to $24 \mathrm{~h}$. HPP-Poloxamer 407 adsorbed nanospheres were collected by spinning down the nanospheres by centrifugation then freeze-drying. Surface coating by HPP-Poloxamer was confirmed by measuring the size and surface zeta potential of the nanospheres in accordance with incubation time.

\section{Nanosphere particle size distribution and surface potential}

Zeta potential and particle size of the nanospheres were measured according to the procedures reported elsewhere. Briefly, solutions containing the above prepared nanospheres were placed into the cuvettes, and their surface charge and particle size determined by measuring electrophoretic mobility using a NICOMP particle and zeta sizer (NICOMP $^{\mathrm{TM}}$ 380ELS, Santa Barbara, CA). All experiments were performed at $25^{\circ} \mathrm{C}, \mathrm{pH} 7.0$ and $677 \mathrm{~nm}$ in wavelength, and a constant angle of $15^{\circ}$. The zeta potential was automatically calculated from the electrophoretic mobility based on Smoluchowski's formula. After determination of the electrophoretic mobility, samples were subject to measurements of the mean particle size using the same equipment, light source, and detection of wavelength. The particle size was reported as the effective mean diameter.

\section{Radioisotope labeling to surface-modified PLGA nanospheres}

\footnotetext{
${ }^{125}$ I was labeled to the surface-modified nanospheres using a modified Chloramine-T method. ${ }^{26}$ Bolton-Huntner
}

agent containing ${ }^{125} \mathrm{I}$ was added to the activated HPP-Poloxamer-coated nanosphere suspension. The labeling efficiency and the radiochemical purity of labeled nanospheres were checked by instant thin-layer chromatography/silica gel (ITLC/SG) according to the manufacturer's instruction. In brief, radioisotope labeling to the nanospheres was detected below the 40-mm line and unlabeled free radioisotopes were detected above the 50-mm line. Labeling efficiency was presented as the ratio of the radioactivity read below $40 \mathrm{~mm}$ to the total radioactivity. To examine the labeling stability of the HPP-Poloxamer 407-coated nanospheres, radioisotopelabeled nanospheres were incubated in human serum for $24 \mathrm{~h}$ at $37^{\circ} \mathrm{C}$ and then radioactivity of pure radioisotopelabeled nanospheres was measured using the same method described above.

\section{Whole body imaging with surface-modified PLGA nanospheres}

Whole body imaging studies were performed using ICR mice weighing 20-25 g and 4-7 weeks old. Mice were imaged at 5-min intervals for an hour after injection of $50 \mu \mathrm{Ci}$ of ${ }^{131}$ I-labeled, HPP-Poloxamer-coated nanosphere suspension into the tail vein. Images were compared with that of ${ }^{99 \mathrm{~m}}$ Tc-labeled tin (Stannous) colloid. All animals were anesthetized by intermittent intravenous administration of anesthetic. A large-field gamma camera fitted with a low-energy and all-purpose collimator allowed visualization of the whole body of the mouse. For the animal experiment, National Institutes of Health $(\mathrm{NIH})$ guidelines for the care and use of laboratory animals (NIH publication no. 85-23 rev. 1985) were observed.

\section{Biodistribution of surface-modified PLGA nanospheres}

Biodistribution experiments were performed using 4-7week-old ICR mice. The mice were injected in the tail vein with ${ }^{125}$ I-labeled HPP-Poloxamer 407-coated nanosphere suspension at $1 \mu \mathrm{Ci}$ of radioactivity. ${ }^{99 \mathrm{~m}} \mathrm{Tc}$-labeled tin (Stannous) colloid with similar particle size distribution and radioactivity was used as a control. Mice were sacrificed after 1, 6, and $24 \mathrm{~h}$. Approximately 0.1-mL blood samples were collected from the abdominal aorta. Whole blood volume of the mice was assumed as $0.05 \mathrm{~mL} / \mathrm{g} .{ }^{27}$ Subsequently, liver, spleen, bone, kidneys, heart, lungs, small intestine, stomach, and muscle were excised. Total radioactivity of blood and each excised organ was measured using a gamma counter and presented as the ratio of percent injected dose $(\%)$ to organ weight $(\mathrm{g})$. For this animal experiment, NIH guidelines for the care and use of laboratory animals $(\mathrm{NIH}$ publication no. 85-23 rev. 1985) were observed.

\section{Statistical analysis}

All measurements were collected in triplicate and expressed as mean \pm standard deviation. One-way analysis of 
variance was used to measure the statistical significance of comparing the biodistribution of the ${ }^{99 \mathrm{~m}} \mathrm{Tc}$-tin colloid and

${ }^{125}$ I-labeled HPP-Poloxamer-coated nanospheres.

\section{RESULTS AND DISCUSSION}

\section{Surface modification of ligand-conjugated Poloxamer 407}

Conjugation of HPP to Poloxamer 407 was confirmed by examining Fourier transform infrared spectra of ester bonds and differential scanning calorimetry (new peak attributed to newly formed ester bond) (data not shown). HPP has a low water solubility; however, our previous research demonstrated conjugated HPP did not significantly affect the hydrophilicity of PEO chain, indicating that hydrophobicity of HPP did not affect the mobility of PEO chain of Poloxamer 407 while permitting radiolabeling ${ }^{125} \mathrm{I}$ or ${ }^{131} \mathrm{I} .{ }^{28}$ It should be pointed out that the rationale to include HPP to the Poloxamer 407 lies in an advantage in efficient radiolabeling ${ }^{125} \mathrm{I}$ to HPP-Poloxamer 407coated nanosphere, while maintaining the steric activity of the Poloxamer 407 on the nanosphere surface.

The uncoated PLGA nanospheres were found to be in the $100 \mathrm{~nm}$ of mean size range required for long circulating and following bone/bone marrow imaging purpose [Fig. 1(a)]. ${ }^{20,21}$ The thickness of the adsorbed layer on the surface of the nanosphere was expected to be related to the concentration of Poloxamer 407 and incubation time. Adsorption of the PEO containing polymers to the hydrophobic nanospheres has been reported to increase nanosphere size because of the formation of a coating layer. ${ }^{29}$ However, HPP-Poloxamer 407-coated nanospheres did not show any significant difference in size distribution from that of original uncoated nanospheres (data not shown), which suggested that coated nanospheres were still able to be utilized as imaging agents. Because the adsorbed layers measured in this study were small compared with the standard deviation of the size measurements, confirmation of nanosphere coating was obtained by measuring the surface potential of the nanospheres. The uncoated nanospheres were highly negatively charged at their surfaces as reflected in their zeta potentials, being attributed to the presence of carboxyl groups on the surface of PLGA. In contrast, the adsorption of Poloxamer 407 to the nanosphere surfaces resulted in decreased surface charge (increased zeta potential), possibly because of the masking of the inherent negative charges of the nanospheres [Fig. 1(b)]. The PEO chains have been known to bring about a shift in the shear plane to a distance further from the nanosphere surface. ${ }^{29}$ The change of surface charge could be related to the incubation time

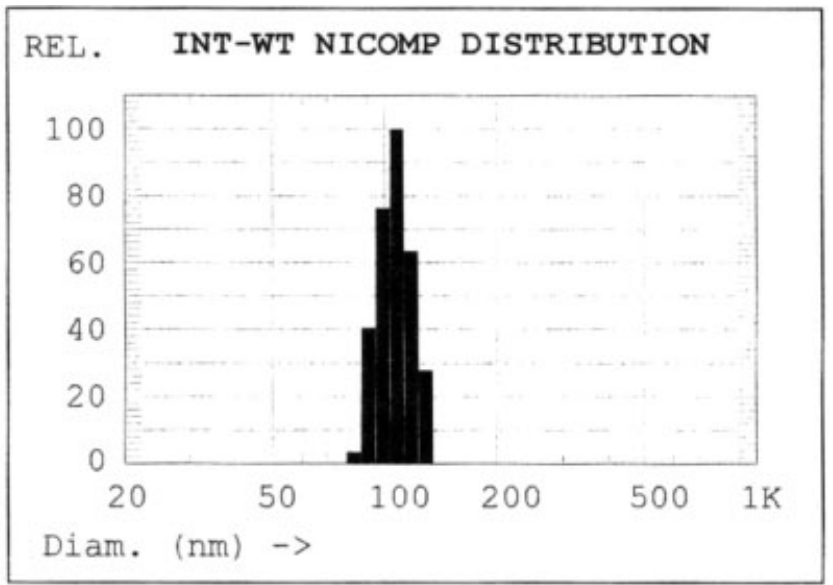

(a)

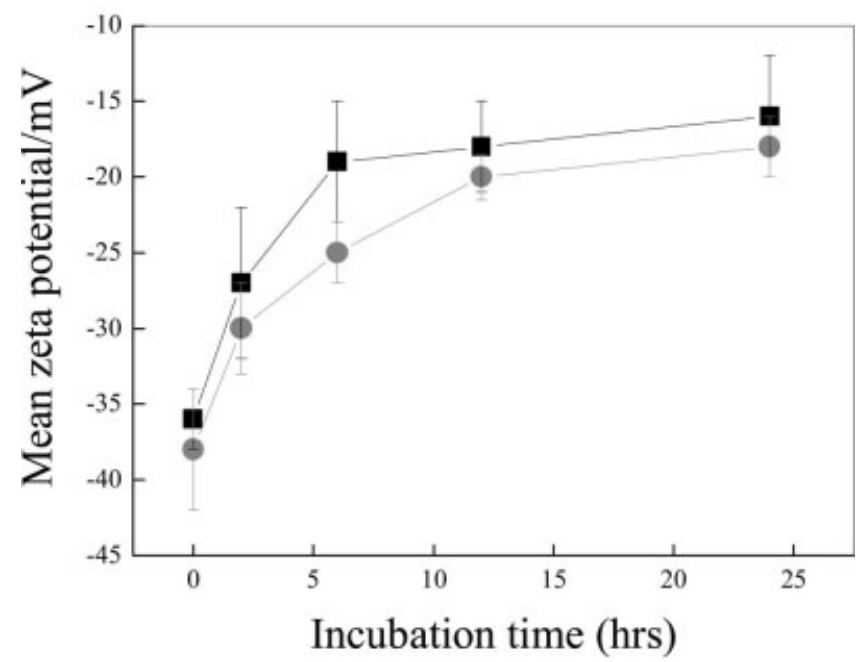

(b)

Figure 1. (a) Dynamic light scattering of PLGA nanospheres shows unimodal size distribution with a mean diameter of $100 \mathrm{~nm}$. (b) Kinetics of the change in the zeta potential of the surface of the nanospheres in accordance with incubation time with the Poloxamer solution; effect of ligand-conjugated Poloxamer on the surface zeta potential. Coating with Poloxamer 407 (ם), and with HPP-Poloxamer 407 (O).

with Poloxamer solution; longer incubation resulted in less surface charge. However, the surface charge reached maximum and was maintained for $24 \mathrm{~h}$, which was attributed to the surface equilibrium of the adsorption. When HPP-Poloxamer 407 solution was applied to the nanospheres, surface charge was decrease as well; however, there was no significant difference from that by an adsorption of Poloxamer 407 solution to the nanospheres, indicating that HPP conjugation did not alter the surface charge of parent Poloxamer 407. The zeta potential results are, therefore, a confirmation of the presence of a coating layer on the nanosphere surface. In addition, even after coating with HPP-Poloxamer 407, the nanospheres 


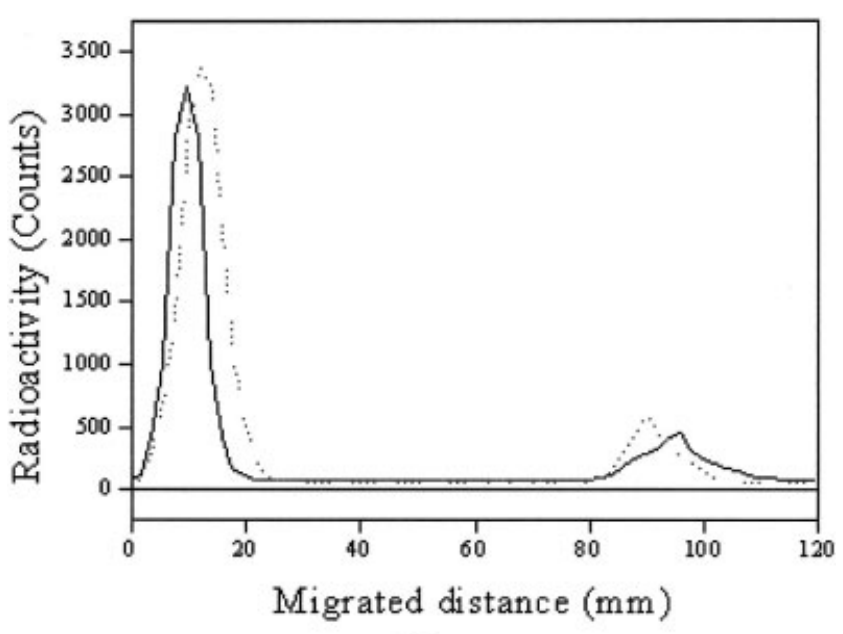

(a)

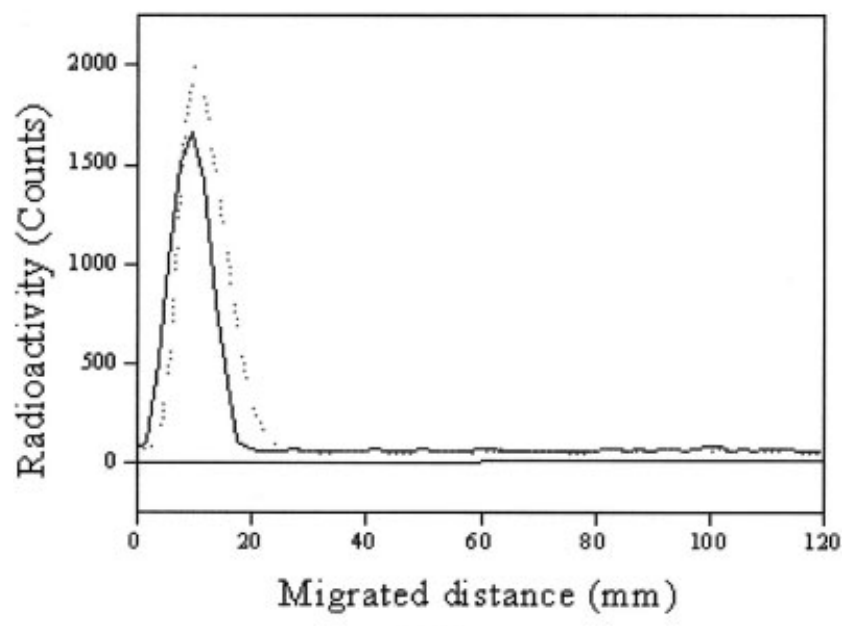

(b)

Figure 2. Labeling efficiency and stability of ${ }^{125} \mathrm{I}$ labeling $(-)$ or ${ }^{131}$ I labeling $(\cdots)$ to nanospheres coated with HPPPoloxamer 407. Labeling efficiency was measured by using ITLC coupled with a radioisotope scanner. Labeled radioisotope to the nanospheres was detected below the $40-\mathrm{mm}$ line whereas free radioisotopes were detected above the 50-mm line. Labeling efficiency (a) and labeling stability (b) of radioisotope to HPP-Poloxamer 407-coated nanospheres are shown as chromatograms.

possessed the size distribution approximately $100 \mathrm{~nm}$ in average, confirming their potential utility as bone imaging agents.

\section{Radioisotope labeling and in vitro stability of HPP-Poloxamer 407-coated nanospheres}

Figure 2(a) shows the ITLC/SG chromatogram demonstrating the labeling efficiency of ${ }^{125} \mathrm{I}$ and ${ }^{131} \mathrm{I}$ to HPP-Poloxamer-coated nanospheres $(10 \mathrm{mg})$, respectively. HPP-Poloxamer 407-coated nanospheres showed a labeling efficiency of $90 \%$, as determined by TLC. Impurities including free isotope were separated from ${ }^{125}$ I-HPP-Poloxamer 407 -coated nanospheres and moved up to the $100-\mathrm{mm}$ region with saline, used as a mobile phase. A single peak of ${ }^{125} \mathrm{I}$-HPP-Poloxamer 407-coated nanospheres was observed at the $10-\mathrm{mm}$ point, indicating that added ${ }^{125} \mathrm{I}$ was almost consumed to label the nanospheres. Figure 2(b) shows the labeling stability of ${ }^{125} \mathrm{I}-$, ${ }^{131} \mathrm{I}$-labeled HPP-Poloxamer 407-coated nanospheres $(5 \mathrm{mg})$, respectively. Because half the amount of nanospheres was utilized in conducting the stability test, overall radioactivity was smaller than that in Figure 2(a), which might seem to be decay of radioactivity during incubation. However, by adjusting the amount of nanospheres, it can be considered that almost the same radioactivity was conserved for 24-h incubation, which is coincident to the general half-life of ${ }^{125} \mathrm{I}$ and ${ }^{131} \mathrm{I}$. In addition, it should be pointed out that only a single peak of ${ }^{125} \mathrm{I}$, or ${ }^{131}$ I-labeled HPP-Poloxamer 407-coated nanosphere was observed, with no other peaks related to dissociated radioisotope. Therefore, radioactive HPPPoloxamer 407-coated nanosphere was considered to be stable in human serum for up to $24 \mathrm{~h}$. High stability of radioisotope labeling may be due to a stable chelation of the isotope to the ligand which is chemically linked to the Poloxamer 407. Stable retention of radioisotope to carrier colloids under physiological condition is essential for measuring in vivo distribution of the colloids. Radioisotopes have been loaded into polymeric nanoparticulates by physical entrapment or direct coupling to polymer chains even in the absence of ligands. ${ }^{24,25,30}$ Physical entrapment of the radioisotopes can incorporate radioisotopes during nanosphere preparation. However, radioisotope at the surface of the nanospheres would be lost in a short time by burst release when injected into the blood stream, resulting in only a small fraction being retained in the nanospheres. Thus, those burst and nonspecific leakage of the radioisotope from the colloid nanospheres to the blood stream result in a biodistribution profile that is not representative of an applied colloid system. Direct coupling may have an advantage in avoiding nonspecific isotope leakage to the blood stream; however, its application is limited because labeling and purification time may exceed the half-life of the radioisotope, which may cause low yield. ${ }^{30}$ Therefore, the direct coupling method is inappropriate for a radioisotope with a short half-life, such as ${ }^{99 \mathrm{~m}} \mathrm{Tc},{ }^{131} \mathrm{I}$, and ${ }^{125} \mathrm{I}$, which are currently used for bone imaging. Polymers synthesized from ${ }^{14} \mathrm{C}$-radiolabeled monomers have been used to prepare radiolabeled nanospheres, for example, ${ }^{14} \mathrm{C}$-labeled polylactic acid nanosphere. ${ }^{31,32}$ The radiolabel is relatively stable because ${ }^{14} \mathrm{C}$ would be released and excreted only after degradation of polymer. However, its application is quite limited because it emits $\beta$-ray, which is not in a sufficient radioactive strength for imaging the target organ. In contrast, our previous and current studies 
demonstrated that PEO-containing copolymers were chemically conjugated to the specific ligands before labeling the desired radioisotope and then radioisotopes, such as ${ }^{99 \mathrm{~m}} \mathrm{Tc},{ }^{125} \mathrm{I}$, and ${ }^{131} \mathrm{I}$, could chelate specifically to the ligand on the polymer. Thus, these ligand-conjugated polymers are ready to carry radioisotope to the target, primarily to the bone and bone marrow. In addition, ITLC data clearly demonstrate the stable retainment of the radioisotope in the HPPPoloxamer 407-coated nanosphere, which enables the prediction of biodistribution image represented by the HPP-Poloxamer 407-coated nanospheres. Overall, the advantage of this system is that it allows easy and stable chelation of radioisotopes to the ligand-polymers in a short time, which permits prompt use for organ imaging.

\section{Whole body imaging}

Figure 3 shows posterior whole body images of mouse after administered with ${ }^{131} \mathrm{I}$-HPP-Poloxamercoated nanospheres. For the whole body imaging, ${ }^{131} \mathrm{I}$ was used because it emits stronger radioactivity for the imaging. As a control, ${ }^{99 \mathrm{~m}}$ Tc-tin colloid with the same size distribution was used. The liver/spleen radioactivity was evident $1 \mathrm{~h}$ after injection of ${ }^{99 \mathrm{~m}} \mathrm{Tc}$-tin colloid [Fig. 3(a)]. ${ }^{99 \mathrm{~m}} \mathrm{Tc}$-antimony, ${ }^{99 \mathrm{~m}} \mathrm{Tc}$-sulfur colloid, and ${ }^{99 \mathrm{~m}} \mathrm{Tc}$-tin colloids are currently investigated imaging agents because they have high sensitivity in bone imaging. However, those colloids are still required to have an improvement in targeting efficiency (currently $0.5-2.7 \%$ uptake by the bone, primarily attributed to the surface hydrophobicity). The whole body image by the ${ }^{131} \mathrm{I}$-HPP-Poloxamer-coated nanospheres was comparable to that of ${ }^{99 \mathrm{~m}} \mathrm{Tc}$-tin colloids [Fig. 3(b)]. Nanospheres coated with ${ }^{131}$ I-HPP-Poloxamer 407 remained largely in the vascular compartment, indicating maintenance in the blood stream, and demonstrated little uptake in the liver/spleen region whereas ${ }^{99 \mathrm{~m}}$ Tc-tin colloids were taken up mainly by liver and spleen. The uptake of the surface-coated nanospheres in the liver and spleen was largely associated with the blood pool in the liver, not with the liver tissue. It is not surprising that surface-modified nanoparticulates with PEO showed more retention in the blood stream while avoiding liver/spleen uptake, as the previous reports already demonstrated. However, our system, which permits rapid and stable radiolabeling without any alteration of PEO chain at the surface, opens possibilities for development of a long circulating and effective bone-targeting imaging agent.

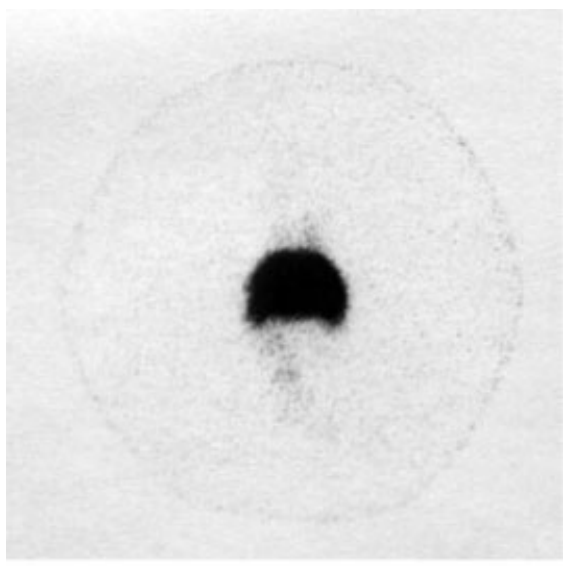

(a)

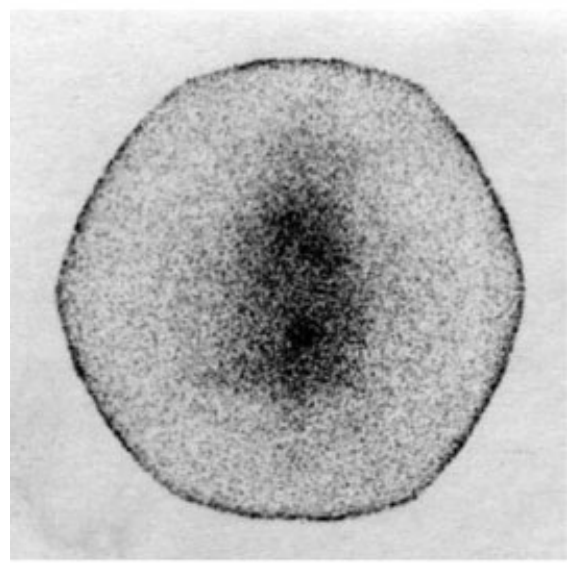

(b)

Figure 3. Whole body scintiscans of mouse $1 \mathrm{~h}$ after intravenous administration of ${ }^{99 \mathrm{~m}} \mathrm{Tc}$-tin colloid (a) and ${ }^{131} \mathrm{I}-\mathrm{HPP}-$ Poloxamer 407-coated nanospheres (b).

\section{Biodistribution of HPP-Poloxamer 407-coated nanospheres}

Figure 4(a) shows the biodistribution of ${ }^{99 \mathrm{~m}} \mathrm{Tc}$-tin colloids in ICR mouse $1 \mathrm{~h}$ after intravenous injection. After $1 \mathrm{~h}$ from injection, most of the injected colloid was found in the liver, spleen, and lung, compared with extremely low uptake by the bone $(1.2 \%)$. This result is quite coincident to that from whole body imaging. When ${ }^{125} \mathrm{I}-\mathrm{HPP}-$ Poloxamer-coated nanospheres were applied to the mouse, the liver and spleen uptake was notably reduced to $11 \%$ and $5 \%$, respectively [Fig. 4(b)]. In contrast, blood retention and bone uptake were significantly increased $(26 \%$ and $15 \%$, respectively). The high blood retainment confirms the result from whole body imaging in Figure 3(b). Compared with that of tin colloid, an increase in amounts of nanospheres remaining in the blood induced their migration to the carcass including bone and bone marrow. The result indicates that these surface-modified nanospheres have proper function of 


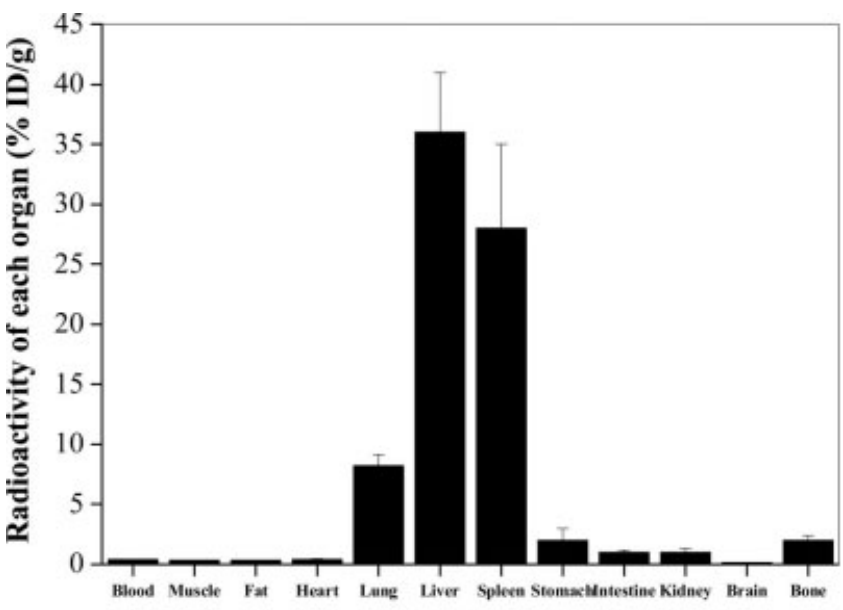

(a)

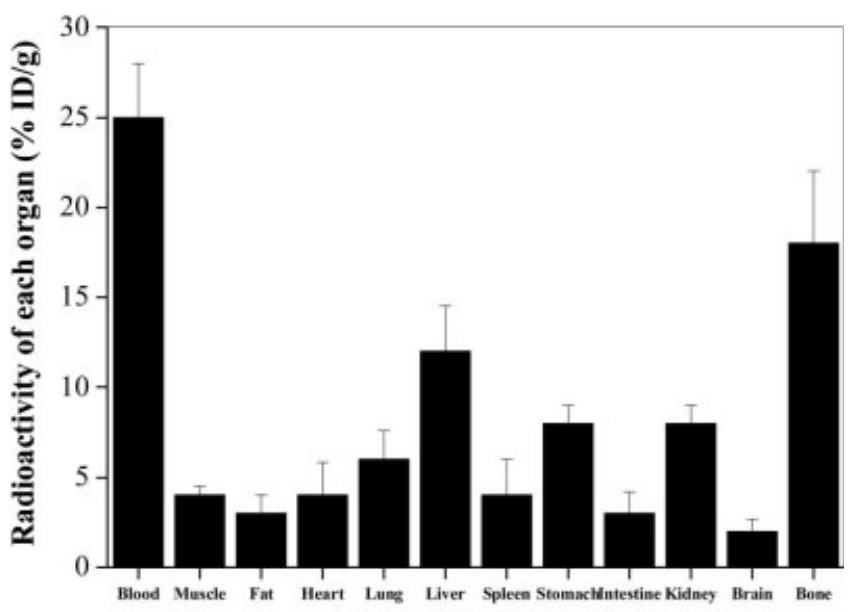

(b)

Figure 4. Distribution of nanocolloids (presented as percent injected dose/whole organ weight) in mouse tissues after intravenous administration of $1 \mu \mathrm{Ci}$ of colloids after $1 \mathrm{~h}$; (a) ${ }^{99 \mathrm{~m}}$ Tc-tin colloids, (b) ${ }^{125} \mathrm{I}$-HPP-Poloxamer-coated nanospheres. Data are presented as mean \pm standard deviation $(n=6)$.

maintaining blood stability and enhancing bone uptake whereas avoiding RES uptake.

The levels of HPP-Poloxamer-coated nanospheres and ${ }^{99 \mathrm{~m}} \mathrm{Tc}$-tin colloids in blood versus time are shown in Figure 5(a). ${ }^{99 \mathrm{~m}} \mathrm{Tc}$-tin colloids showed lower blood retention than HPP-Poloxamer-coated nanospheres for the observation period. Even at $24 \mathrm{~h}$, HPP-Poloxamer-coated nanospheres showed notably higher retainment in the circulation than ${ }^{99 \mathrm{~m}} \mathrm{Tc}$-tin colloid. HPP-Poloxamer-coated nanospheres exhibited $17 \%$, $13 \%$ of the decrease from the original dose at $6 \mathrm{~h}$ and $24 \mathrm{~h}$ in the blood, respectively. Thus, prolonged retention of HPP-Poloxamer-coated nanospheres indicates that the surface layer of HPP-Poloxamer at the nanospheres stably persisted in the blood.

PEO is generally known to suppress protein and cellular adsorption at the surfaces and interfaces by its hydrophilicity and chain mobility, and to prolong the circulation time of the nanoparticles including liposomes. ${ }^{22,23,33-35}$ It was not surprising that the Poloxamer-modified nanospheres showed the reduced uptake by the RES in the liver or spleen, which was accompanied by extended blood circulation times [Fig. 5(b,c)]. The ability of PEO-modified drug carriers in avoiding the RES is in sharp contrast with most unmodified nanocolloids, which are rapidly cleared from the circulation within minutes. The Poloxamercoated nanospheres clearly demonstrated elevated plasma levels in accordance with time when compared with ${ }^{99 \mathrm{~m}} \mathrm{Tc}$-tin colloids. Our hypothesis was that such high retention of particles could result in the enhanced uptake by bone and bone marrow, which is another big RES after liver or spleen. The exact mechanism of bone and bone marrow uptake remains to be elucidated; however, our previous and current studies could propose a responsible mechanism-nano-sized colloid particles leave the circulation and can be captured by the liver and spleen. The remaining particles in the blood stream could enter the extravascular spaces of the bone and bone marrow with discontinuity of the vascular endothelium and basement membrane. ${ }^{28,36,37}$ Bone uptake of the HPP-Poloxamercoated nanospheres was markedly higher than that of ${ }^{99 \mathrm{~m}} \mathrm{Tc}$-tin colloids up to $24 \mathrm{~h}$, corroborating that these surface-modified nanospheres had proper function of enhancing bone uptake while avoiding RES uptake [Fig. 5 (d)]. The interaction between the surface of the bone marrow and hydrophilic PEO chain including Poloxamer has not been clearly established; however, several hypotheses for the recognition mechanism have been suggested. ${ }^{36-40}$ Plasma components, such as erythropoietin, transferrin, transcobalamine, or endothelial-derived factor, or cellular adhesion molecule such as lectin, have been proposed to mediate the recognition of PEO-anchored surface by the bone marrow. ${ }^{36-40}$ When adsorbed onto the surface of Poloxamer-coated nanospheres, those above recognizable plasma molecules may exhibit the specificity for certain microdomains on the bone marrow endothelial cell surface, which is similar to the uptake of surfacesugar bearing particles by the hepatocytes in the liver. Therefore, the nanoparticles with surface PEO by adsorption of Poloxamer can be favorable in improving blood compatibility by its hydrophilicity, protecting from RES uptakes, being recognized by the bone marrow, hence final enhancement in the bone uptake.

As one of the requirements for an efficient bone imaging agent, proper excretion may be suggested. ${ }^{14}$ HPP-Poloxamer-coated nanospheres initially showed much less uptake by the other organs than liver or spleen. In addition, the radioactivity significantly reduced after $24 \mathrm{~h}$, which provided the evidence of proper excretion of these nanospheres [Fig. 5(e)]. It should be pointed out that our system has a unique 


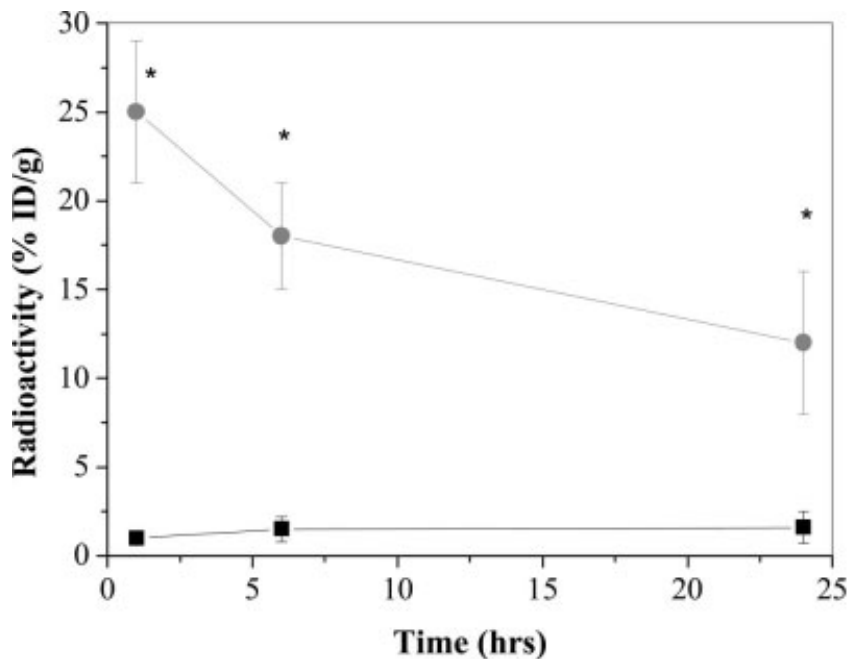

(a)

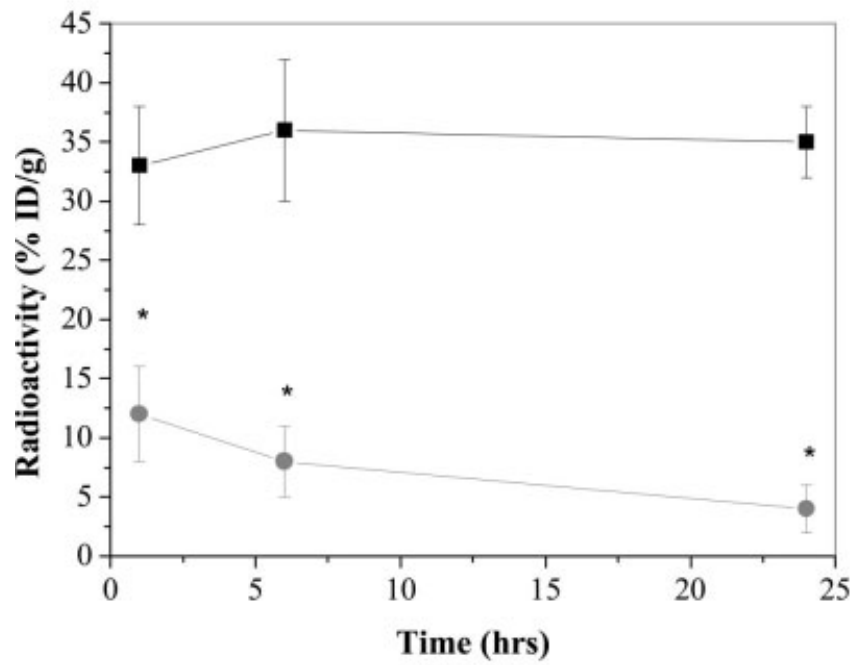

(b)

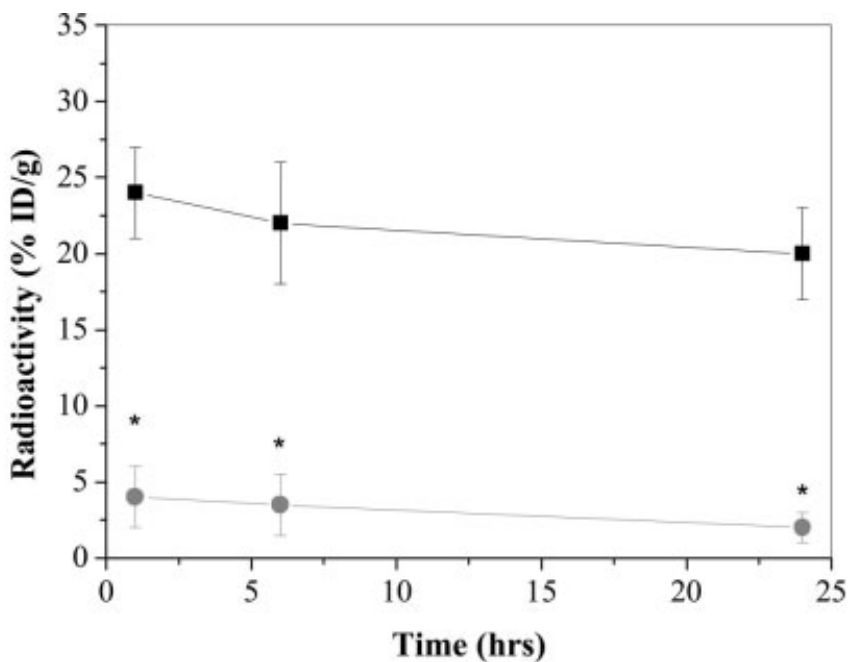

(c)

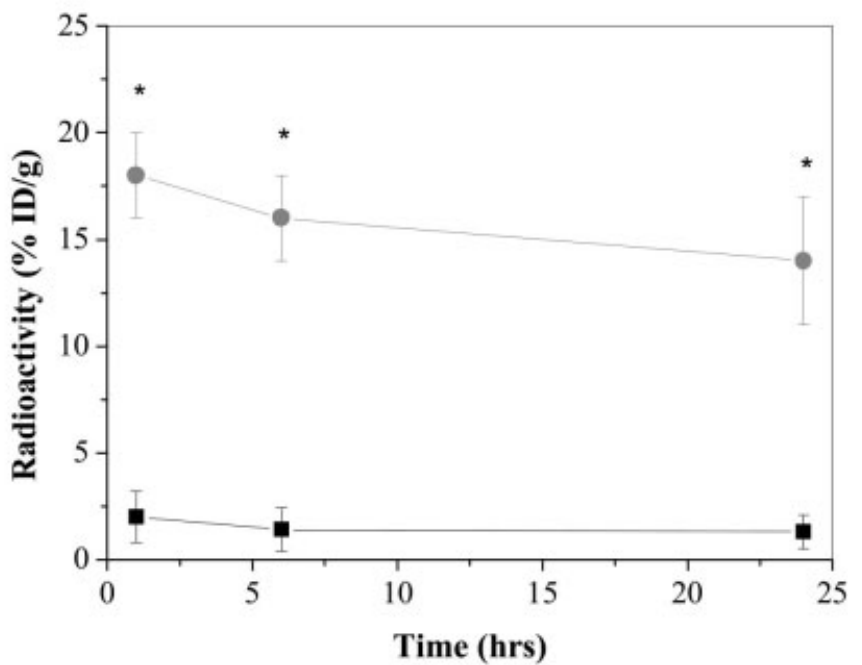

(d)

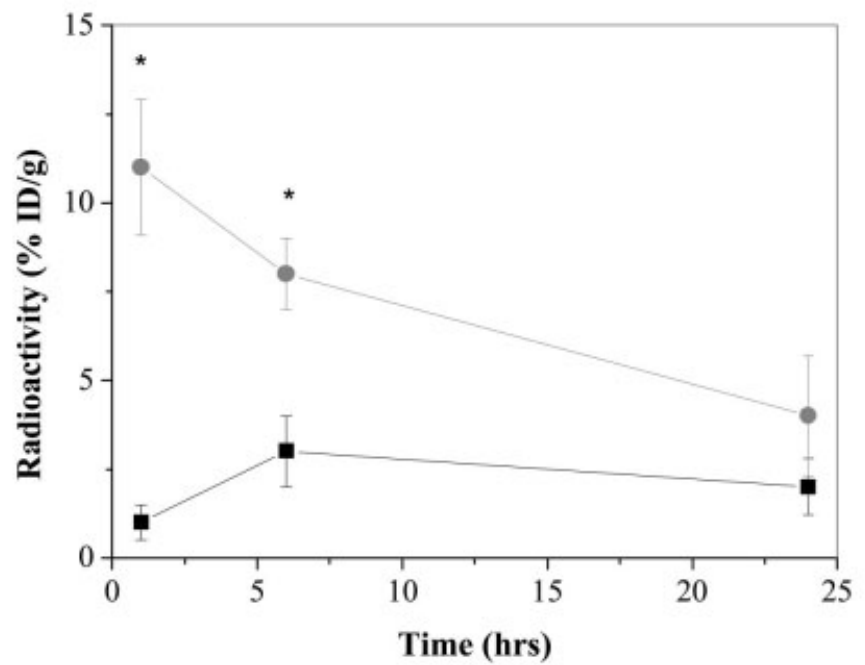

(e)

Figure 5. Levels of ${ }^{99 m}$ Tc-tin colloids $(\square)$ and ${ }^{125} \mathrm{I}-\mathrm{HPP}-$ Poloxamer-coated nanospheres $(\boldsymbol{)})$ in the blood (a), liver (b), spleen (c), bone (d), and the kidney (e) in accordance with time. Data are presented as mean \pm standard deviation $(n=6)$. ${ }^{*} p<0.05$, as compared with that of ${ }^{99 m}$ Tc-tin colloids. 
advantage in labeling radioisotope to the polymeric colloids via chelation to the ligand which is already conjugated to the polymeric colloid surface. This method could offer a rapid and stable radiolabeling to the colloids whereas maintaining the original behavior of the colloids in the body. Whole body imaging study indicates that the use of a more sensitive radioisotope such as ${ }^{99 \mathrm{~m}} \mathrm{Tc},{ }^{111} \mathrm{In}$ can provide an enhanced imaging.

Overall, the HPP-Poloxamer-coated PLGA nanospheres might be suggested as an efficient bone and bone marrow imaging agent while overcoming the current limitations in developing bone marrow imaging agents. Optimization of radioisotope labeling to ligand-polymer, and related bone and bone marrow uptake research is now in progress in our laboratory.

\section{CONCLUSION}

Radioisotope carrying, surface-modified PLGA nanospheres were prepared for targetable bone and bone marrow imaging. The nanospheres were coated by ligand-conjugated Poloxamer 407 to provide the nanospheres with the surface hydrophilicity due to PEO segment and ease of radiolabeling. These nanospheres had proper size distribution and hydrophilicity for effective bone imaging. The ligand-conjugated Poloxamer 407-coated nanospheres showed higher and stable radioisotope labeling efficiency. In addition, radioisotope-carrying nanospheres demonstrated increased blood circulation followed by bone uptake. Therefore, the Poloxamer 407-coated nanospheres, which carryies radioisotope might be a potential tool for effective bone and bone marrow imaging.

\section{References}

1. Mudun A, Unal S, Aktay R, Akmehmet S, Cantez S. Tc-99m nanocolloid and Tc-99m MDP three-phase bone imaging in osteomyelitis and septic arthritis. Clin Nucl Med 1995;20(9):772778.

2. Seabold JE, Nepola JV, Marsh JL, Hawes DR, Justin EP, Ponto JA, Pettit WA, el-Khoury GY, Kirchner PT. Postoperative bone marrow alterations: Potential pitfalls in the diagnosis of osteomyelitis with In-111-labeled leukocyte scintigraphy. Radiology 1991;180(3):741-747.

3. Alexlsson B, Kalin B, Von Krusenstierna S, Jacobsson H. Comparison of In-111 granulocytes and Tc-99m albumin colloid for bone marrow scintigraphy by the use of quantitative SPECT imaging. Clin Nucl Med 1990;15(7):473-479.

4. Widding A, Smolorz J, Franke M, Linden A, Diehl V, Schicha H. Bone marrow investigation with technetium-99m microcolloid and magnetic resonance imaging in patients with malignant myelolympho-proliferative diseases. Eur J Nucl Med 1989;15(5): $230-238$
5. Southee AE, Lee KJ, Rossleigh MA, Morris JG. Extensive pelvic infarction diagnosed by radionuclide skeletal and bone marrow imaging. 1988;13(8):613-614.

6. Jones RJ. The role of bone marrow imaging. Radiology 1992; 183(2):321-322.

7. Balaban EP, Walker BS, Cox JV, Sein AA, Abrams PG, Salk D, Sheenhan RG, Frenkel EP. Radionuclide imaging of bone marrow metastases with a Tc-99m labeled monoclonal antibody to small cell lung carcinoma. Clin Nucl Med 1991;16(10):732-736.

8. Datz FL, Taylor A Jr. The clinical use of radionuclide bone marrow imaging. Semin Nucl Med 1985;15(3):239-259.

9. Graeb D. Role of MRI bone marrow imaging in recurrent lymphoma. Can Assoc Radiol J 1990;41(1):51.

10. Ishimura J, Fukuchi M. Scintigraphic evaluation of secondary myelofibrosis associated with prostatic cancer before and after hormone therapy. Clin Nucl Med 1990;15(5):330-333.

11. Desai AG, Thakur ML. Radiopharmaceuticals for spleen and bone marrow studies. Semin Nucl Med 1985;15:229-238.

12. Edward CL, Sitterson GA, Knisely RM. Clinical bone marrow scanning with radioisotopes. Blood 1964;23:741-756.

13. Illum L, Davis SS. Targeting of colloidal particles to the bone marrow. Life Sci 1987;40:1553-1560.

14. Blend MJ, Pavel DG. Bone marrow imaging: A comparison study using a 99Tcm-sulphur colloid versus a new 99Tcmmicroaggregated albumin. Nucl Med Commun 1986;7(10):787789 .

15. Bulte JWM, de Cuyper M, Despres D, Frank JA. Short- vs. long-circulating magnetoliposomes as bone marrow-seeking MR contrast agents. J Magn Reson Imaging 1999;9:329-335.

16. Hertzberg C, Orlic D. An electron microscopic study of ethythrophagocytosis in the fetal and neonatal rabbit. J Reticuloendothel Soc 1980;28:15-26.

17. Hussain MM, Mahley RW, Boyless JK, Lindquist PA, Brecht WJ, Innerarity TL. Chylomicron metabolism. J Biol Chem 1989;264(30): 17931-17938.

18. Okuhata Y. Delivery of diagnostic agents for magnetic resonance imaging. Adv Drug Deliv Rev 1999;37:121-137.

19. Hamm B, Staks T, Taupitz M, Maibauer R, Speidel A, Huppertz A, Frenzel T, Lawaczeck R, Woff KJ, Lange L. Contrast-enhanced MR imaging of liver and spleen: First experience in humans with a new superparamagnetic iron oxide. J Magn Reson Imaging 1994;4:659-668.

20. Arturson P, Laakso T, Edman P. Acrylic microspheres in vivo. IX. Blood elimination kinetics and organ distribution in microparticles with different surface characteristics. J Pharm Sci 1983; 72:1415-1420.

21. Vandorpe J, Schacht E, Dunn S, Hawley A, Stolinik S, Davis SS, Garnett MC, Davies MC, Illum L. Long circulating biodegradable poly(phosphazene) nanoparticles surface modified with poly(phosphazene)-poly(ethylene oxide) copolymer. Biomaterials 1997; 18:1147-1152

22. Porter CJH, Moghimi SM, Illum L, Davis SS. The polyoxyethylene/polyoxypropylene block co-polymer Poloxamer-407 selectively redirects intravenously injected microspheres to sinusoidal endothelial cells of rabbit bone marrow. FEBS Lett 1992; 305(1):62-66

23. Harper CJ, Davies MC, Davis SS, Tadros TF, Irving MP, Waters JA. Steric stabilization of microspheres with grafted polyethylene oxide reduces phagocytosis by rat Kupffer cells in vitro. Biomaterials 1991;12:695-700.

24. Hawley AE, Illum L, Davis SS. Lymph node localization of biodegradable nanospheres surface modified with poloxamer and poloxamine block copolymers. FEBS Lett 1997;400:319-323.

25. Hazrati AM, Akrawi S, Hickey AJ, Wedlund P, Macdonald J, DeLuca PP. Tissue distribution of Indium-111 labeled poly(glycolic acid) matrices following jugular and hepatic portal vein administration. J Control Release 1989;9:205-214. 
26. Chen P, Hussain A, Tai HH, Dittert LW. An improved method of radioiodination with chloramines T. Anal Biochem 1994; 219(1):159-161.

27. Liu F, Song YK, Liu D. Hydrodynamics-based transfection in animals by systemic administration of plasmid DNA. Gene Ther 1999;6:1258-1266.

28. Park YJ, Lee JY, Chang YS, Jeong JM, Chung JK, Lee MC, Park KB, Lee SJ. Radioisotope carrying polyethylene oxide-polycaprolactone copolymer micelles for targetable bone imaging. Biomaterials 2002;23:873-879.

29. Hawley AE, Illum L, Davis SS. Preparation of biodegradable, surface engineered PLGA nanospheres with enhanced lymphatic drainage and lymph node uptake. Pharm Res 1997;14(5): 657-661.

30. Yeates DB, Warbich A, Aspin A. Production of 99mTc labeled albumin microspheres for lung clearances studies for inhalation scanning. Int J Appl Radiat Isot 1974;25:578-583.

31. Bazile DV, Robert C, Huve P, Verrecchia T, Marlard M, Fryman A, Veilard M, Spenlehauer G. Body distribution of fully biodegradable [14C]-poly(lactic acid) nanoparticles coated with albumin after parenteral administration to rats. Biomaterials 1992; 13(15):1093-1102.

32. Landry FB, Bazile DV, Spenlehauer G, Veillard M, Kreuter J. Peroral administration of 14C-poly(D,L-lactic acid) nanoparticles coated with human serum albumin or polyvinyl alcohol to guinea pigs. J Drug Target 1998;6(4):293-307.

33. Illum L, Davis SS, Muller RH, Mak E, West P. The organ distribution and circulation time of intravenously injected col- loidal carriers sterically stabilized with block copolymer poloxamine 908. Life Sci 1987;40:367-374.

34. Moghimi SM, Hawley AE, Christy NM, Gray T, Illum L, Davis SS. Surface engineered nanospheres with enhanced drainage into lymphatics and uptake by macrophages of the regional lymph nodes. FEBS Lett 1994;344(1):25-30.

35. Moghimi SM, Muir IS, Illum L, Davis SS. Coating particles with a block copolymer (poloxamine 908) suppresses opsonization but permits the activity of dysopsonins in the serum. Biochim Biophys Acta 1986;1179(2):157-165.

36. Moghimi SM, Illum L, Davis SS. Physiopathological and physicochemical considerations in targeting of colloids and drug carriers to the bone marrow. Crit Rev Ther Drug Carrier Syst 1990;7(3):187-209.

37. Moghimi SM, Patel HM. Opsonophagocytosis of liposomes by peritoneal macrophages and bone marrow reticuloendothelial cells. Biochim Biophys Acta 1992;1135(3):269-274.

38. Hardy CL, Megason GC. Specificity of hematopoietic stem cell homing. Hematol Oncol 1996;14(1):17-27.

39. Liu ZY, Gangju RK, Wang JF, Schweitzer K, Weksler B, Avraham S, Groopman JE. Characterization of signal transduction pathways in human bone marrow endothelial cells. Blood 1997; 90(6):2253-2259.

40. Asosingh K, Gunthert U, De Raeve H, Van Riet IV, Van Camp $\mathrm{BV}$, Vanderkerken K. A unique pathway in the homing of murine multiple myeloma cells: CD44v10 mediates binding to bone marrow endothelium. Cancer Res 2001;61:2862-2865. 